\title{
On Cotorsion Images of the Baer-Specker Group
}

\author{
B. GOLDSMITH, T. KELLY AND S. L. WALLUTIS
}

A paper in the Bulletin [1] some years ago has discussed in detail the so-called Baer-Specker group $P$, the full Cartesian product of countably many copies of the integers, $\mathbb{Z}$. Despite its seemingly straightforward presentation as $P=\prod_{i \in \omega} \mathbb{Z} e_{i}=\mathbb{Z}^{\omega}$, this group has a very rich structure of subgroups: on the one hand it is an $\aleph_{1}$-free group (i.e., all its countable subgroups are free Abelian groups — see [4, Theorem 19.2]), but on the other hand it also possesses subgroups which exhibit a high degree of pathological behaviour. There is, for example, a subgroup $G$ of $P$ with the property that $G \cong G \oplus G \oplus G$ but $G \not G \oplus G$ - see [2].

The objective of the current paper is to consider the epimorphic images of $P$ and to show that these are classifiable in a very clear way, but that the possible images are very wide-ranging.

Before establishing the desired results we recall some standard notation and concepts. All groups will be additively written Abelian groups and the standard reference for related concepts is the twovolume work of Fuchs [4]. The subgroup of $P$ consisting of the restricted product (or direct sum) will be denoted by $S$, that is $S=\bigoplus_{i \in \omega} \mathbb{Z} e_{i}=\mathbb{Z}^{(\omega)}$. The letter $\mathbb{Q}$ will denote the additive group of rationals, $\mathbb{Z}\left(p^{k}\right)$ the cyclic group of order $p^{k}, \mathbb{Z}\left(p^{\infty}\right)$ the Prüfer quasi-cyclic group of all complex $p^{n}$-th $(n \in \omega)$ roots of unity and $J_{p}$ the additive group of $p$-adic integers. This latter may be regarded as the group of all formal sums $\sum_{n=0}^{\infty} a_{n} p^{n}$ where the coefficients $a_{n}$ satisfy $0 \leq a_{n}<p$ and addition is carried out componentwise with the usual 'carry' operation.

The division of Abelian groups into reduced groups and groups with a non-trivial divisible part is fundamental. Recall that an Abelian group $D$ is said to be divisible if, given any element $x \in D$ and integer $n$, there is an element $y \in D$ such that $x=n y$. A divisible group is known to be of the form $\bigoplus \mathbb{Z}\left(p^{\infty}\right)^{\left(\alpha_{p}\right)} \oplus \mathbb{Q}^{(\alpha)}$ for $p$ prime 
some cardinals $\alpha, \alpha_{p}$, where $G^{(\kappa)}$ means the direct sum of $\kappa$ copies of $G$. The divisible groups are precisely the injective objects in the category of Abelian groups and, consequently, it can be shown that any $G$ can be written as $G=D \oplus H$, where $D$ is divisible and $H$ has no divisible subgroups; a group such as $H$ is said to be reduced.

We shall also require the well-known Cartan-Eilenberg sequences from homological algebra, but only in the following simplified versions: Given a short exact sequence

$$
0 \rightarrow A \rightarrow B \rightarrow C \rightarrow 0
$$

and arbitrary groups $X, Y$ the following sequences are also exact:

$$
\begin{aligned}
0 \rightarrow \operatorname{Hom}(X, A) & \rightarrow \operatorname{Hom}(X, B) \rightarrow \operatorname{Hom}(X, C) \\
& \rightarrow \operatorname{Ext}(X, A) \rightarrow \operatorname{Ext}(X, B) \rightarrow \operatorname{Ext}(X, C) \rightarrow 0
\end{aligned}
$$

and

$$
\begin{aligned}
0 \rightarrow \operatorname{Hom}(C, Y) & \rightarrow \operatorname{Hom}(B, Y) \rightarrow \operatorname{Hom}(A, Y) \\
& \rightarrow \operatorname{Ext}(C, Y) \rightarrow \operatorname{Ext}(B, Y) \rightarrow \operatorname{Ext}(A, Y) \rightarrow 0 .
\end{aligned}
$$

Here we are writing, as usual, $\operatorname{Hom}(X, A)$ for the group of homomorphisms from $X$ into $A$ and $\operatorname{Ext}(X, A)$ for the group of extensions of $A$ by $X$.

The notion of algebraic compactness is due to Kaplansky and originated in his study of the algebraic structure of compact Abelian groups. There are many equivalent definitions of the concept, but for our purposes it will suffice to say that the group $G$ is algebraically compact if, and only if it has the form $G=D \oplus H$, where $D$ is divisible and $H$ is reduced and complete in its $\mathbb{Z}$-adic topology, i.e., the linear topology having the subgroups $n H(n \in \mathbb{Z}, n \neq 0)$ as a basis of neighbourhoods of zero. It is, in fact, possible to give a complete description of reduced algebraically compact groups. Such a group $A$ has the form $A=\prod_{p \text { prime }} A_{p}$ where each $A_{p}$ is the $p$-adic completion of $\left(J_{p}^{\left(m_{0}\right)} \oplus \bigoplus_{k=1}^{\infty} \mathbb{Z}\left(p^{k}\right)^{\left(m_{k}\right)}\right)$ for some arbitrary cardinal numbers $m_{0}, m_{k}$. These cardinals form a complete, independent system of invariants for $A$ - details may be found in [4, Vol I, pp. 167-169].

The final concept we need is the notion of a group being cotorsion. This fundamentally important concept in group and module 
theory was introduced by Harrison in the 1960s and is given by a homological definition.

Definition 1. A group $G$ is said to be cotorsion if $\operatorname{Ext}(\mathbb{Q}, G)=0$.

For torsion-free groups cotorsion coincides with algebraic compactness while a torsion cotorsion group has a simple structure: it is a direct sum of a divisible group and a bounded group. Despite the seeming simplicity of these two cases, cotorsion groups may be quite complex and, in fact, a group is cotorsion if, and only if it is an epimorphic image of an algebraically compact group — see [4, Proposition 54.1].

It is the aim of this paper to show that any cotorsion group of cardinality at most $2^{\aleph_{0}}$ is an epimorphic image of the algebraic compact group $P / S$ and hence of the Baer-Specker group $P$. Before we prove this main result (Theorem 7 ) we determine the structure of $P / S$ (Proposition 5). First we need:

Lemma 2. If $C$ is a reduced cotorsion group then there is a natural isomorphism $\operatorname{Ext}(\mathbb{Q} / \mathbb{Z}, C) \cong C$.

Proof. Let $C$ be a reduced cotorsion group and consider the exact sequence

$$
0 \rightarrow \mathbb{Z} \rightarrow \mathbb{Q} \rightarrow \mathbb{Q} / \mathbb{Z} \rightarrow 0
$$

This induces a Cartan-Eilenberg sequence

$$
\begin{aligned}
0 \rightarrow \operatorname{Hom}(\mathbb{Q} / \mathbb{Z}, C) \rightarrow \operatorname{Hom}(\mathbb{Q}, C) \rightarrow \operatorname{Hom}(\mathbb{Z}, C) & \\
& \rightarrow \operatorname{Ext}(\mathbb{Q} / \mathbb{Z}, C) \rightarrow \operatorname{Ext}(\mathbb{Q}, C) \rightarrow \operatorname{Ext}(\mathbb{Q} / \mathbb{Z}, C) \rightarrow 0 .
\end{aligned}
$$

However, $\operatorname{Hom}(\mathbb{Q}, C)=0$ since $C$ is reduced, $\operatorname{Ext}(\mathbb{Q}, C)=0$ as $C$ is cotorsion and $\operatorname{Hom}(\mathbb{Z}, C)$ is naturally isomorphic to $C$. Thus we obtain $C \cong \operatorname{Hom}(\mathbb{Z}, C) \cong \operatorname{Ext}(\mathbb{Q} / \mathbb{Z}, C)$ as required.

Lemma 3. The quotient group $P / S$ is algebraically compact.

Proof (Sketch). It is possible, using standard topological arguments, to reduce the problem to showing that every neat Cauchy sequence in $P / S$ has a limit in $P / S$. So suppose $\left\{x_{n}+S\right\}_{n \in \omega}$ is a neat Cauchy sequence, i.e., $x_{n+1}-x_{n} \equiv n y_{n} \bmod S$ for some $y_{n} \in P$. Clearly, since we are working modulo $S$, we may choose representatives $y_{n}$ in such a way that the first $(n-1)$ components of $y_{n}$ (as a 'vector' in $P)$ are zero. Notice, that this means that an infinite sum $\sum_{n=0}^{\infty} n y_{n}$ makes sense in $P$ since the summation is finite on each component. 
It is now straightforward to check that $x+S=\left(x_{0}+\sum n y_{n}\right)+S$ is the desired limit.

Lemma 4. There exists a divisible subgroup of $P / S$ of power the continuum, $2^{\aleph_{0}}$.

Proof. For each function $\varepsilon: \omega \rightarrow 2=\{0,1\}$, we define elements $x_{\varepsilon}^{m} \in P(m \in \omega)$ by

$$
x_{\varepsilon}^{m}=(\underbrace{0, \ldots, 0}_{m \text { times }}, m ! \varepsilon(m),(m+1) ! \varepsilon(m+1), \ldots)
$$

and let

$$
x_{\varepsilon}=x_{\varepsilon}^{0}=(\varepsilon(0), \varepsilon(1), 2 \varepsilon(2), \ldots) .
$$

Then $x_{\varepsilon}$ is congruent to $x_{\varepsilon}^{m}$ modulo $S$ and thus divisible by $m$ ! for each $m \in \omega$. Hence the subgroup $D$ of $P / S$ generated by the cosets $x_{\varepsilon}+S(\varepsilon: \omega \rightarrow 2)$ is divisible.

Now, two cosets $x_{\varepsilon}+S, x_{\delta}+S(\varepsilon, \delta: \omega \rightarrow 2)$ coincide if and only if the set $\{m \in \omega \mid \varepsilon(m) \neq \delta(m)\}$ is finite. There are, however, $2^{\aleph_{0}}$ infinite subsets of $\omega$ such that the intersection of any two of them is finite. Consequently, there are $2^{\aleph_{0}}$ mappings from $\omega$ to 2 which differ in infinitely many values, since any subset $X$ of $\omega$ induces a map $\varepsilon: \omega \rightarrow 2$ with $\varepsilon(m)=0$ for $m \notin X$ and $\varepsilon(m)=1$ for $m \in X$.

Therefore the divisible subgroup $D$ of $P / S$ defined above has cardinality $2^{\aleph_{0}}$.

Proposition 5. $P / S$ is of the form $\mathbb{Q}^{\left(2^{\aleph_{0}}\right)} \oplus \prod_{p} \widehat{J_{p}^{\left(2^{\aleph_{0}}\right)}}$, where $\widehat{J_{p}^{\left(2^{\aleph_{0}}\right)}}$ is the $p$-adic completion of $J_{p}^{\left(2^{\aleph_{0}}\right)}=\bigoplus_{2^{\aleph_{0}}} J_{p}$.

Proof. $P / S$ is algebraically compact by Lemma 3 . Using the Structure Theorem for Reduced Algebraically Compact Groups and the fact that $P / S$ is torsion-free, we have that $P / S \cong \mathbb{Q}^{(\delta)} \oplus \prod_{p \text { prime }} \widehat{J_{p}^{\left(\beta_{p}\right)}}$ for some cardinals $\delta$ and $\beta_{p}$. We have already seen in Lemma 4 that $\delta=2^{\aleph_{0}}$ and so it remains to establish the precise structure of the reduced part $R / S=\prod_{p \text { prime }} \widehat{J_{p}^{\left(\beta_{p}\right)}}$ of $P / S$, i.e., we wish to show that $\beta_{p}=2^{\aleph_{0}}$ for all primes $p$. 
First we consider $R / S$ modulo $q(R / S)$ for some fixed prime $q$ :

$$
\begin{aligned}
\prod_{p} \widehat{J_{p}^{\left(\beta_{p}\right)}} / q \prod_{p} \widehat{J_{p}^{\left(\beta_{p}\right)}} & \cong \widehat{J_{q}^{\left(\beta_{q}\right)}} / q \widehat{J_{q}^{\left(\beta_{q}\right)}} \cong J_{q}^{\left(\beta_{q}\right)} / q J_{q}^{\left(\beta_{q}\right)} \\
& \cong\left(J_{q} / q J_{q}\right)^{\left(\beta_{q}\right)} \cong(\mathbb{Z} / q \mathbb{Z})^{\left(\beta_{q}\right)} .
\end{aligned}
$$

Hence $\beta_{q}$ coincides with the cardinality of $(R / S) /(q(R / S))$ since $\mathbb{Z} / q \mathbb{Z}$ is finite. Moreover, note that $(R / S) /(q(R / S))$ is naturally isomorphic to $(P / S) /(q(P / S))$. Therefore it will be sufficient to show that $(P / S) /(q(P / S))$ contains $2^{\aleph_{0}}$ different elements in order to obtain $\beta_{q}=2^{\aleph_{0}}$ for any prime $q$.

Now, for any $\varepsilon: \omega \rightarrow 2$ we define an element $x_{\varepsilon} \in P$ by

$$
x_{\varepsilon}=(\varepsilon(0), \varepsilon(1), \ldots, \varepsilon(m), \ldots) .
$$

As already discussed in the proof of Lemma 4 there exist $2^{\aleph_{0}}$ mappings $\omega \rightarrow 2$ which differ in infinitely many values. Hence, for two such functions $\varepsilon, \delta: \omega \rightarrow 2$, we have $x_{\varepsilon}(m)-x_{\delta}(m) \in\{-1,1\}$ for infinitely many $m$ 's. Thus it follows immediately that the two cosets $x_{\varepsilon}+S, x_{\delta}+S$ are different elements of $P / S$ which cannot be congruent modulo $q(P / S)$ since $x_{\varepsilon}-x_{\delta}+S \notin q(P / S)$.

Therefore $\beta_{q}=|(P / S) /(q(P / S))|=2^{\aleph_{0}}$ and this completes the proof.

The motivation for our main result comes from a deep theorem of Nunke - see e.g. [3, Chapter IX] — which gives a vital first characterization of the images of the Baer-Specker group $P$.

Theorem 6 (Nunke). Every epimorphic image $G$ of $P$ has the form $G=P_{1} \oplus C$ where $P_{1}$ is a Cartesian product of countably many copies of $\mathbb{Z}$ and $C$ is a cotorsion group.

Our main result extends this theorem of Nunke by showing that, in essence, every possible cotorsion group actually appears as an image of $P$.

Main Theorem 7. If $C$ is a cotorsion group of cardinality at most $2^{\aleph_{0}}$, then $C$ is an epimorphic image of the Baer-Specker group $P$.

Proof. Let $C$ be a cotorsion group with $|C| \leq 2^{\aleph_{0}}$. We show that $C$ is an epimorphic image of $P / S \cong \mathbb{Q}^{\left(2^{\aleph_{0}}\right)} \oplus \prod_{p \text { prime }} \widehat{J_{p}^{\left(2^{\aleph_{0}}\right)}}$. It is then immediate that $C$ is also an epimorphic image of $P$. 
As mentioned before we have $C=C^{\prime} \oplus D$ with $C^{\prime}$ reduced and $D$ divisible. Since $|D| \leq|C| \leq 2^{\aleph_{0}}$ it is hence obvious that $D$ is an epimorphic image of $\mathbb{Q}^{\left(2^{\aleph_{0}}\right)}$.

Thus we may proceed to consider $C$ to be a reduced cotorsion group. Let $\mathcal{D}(C)$ be the divisible hull of $C$ and consider the short exact sequence

$$
0 \rightarrow C \rightarrow \mathcal{D}(C) \rightarrow \mathcal{D}(C) / C \rightarrow 0
$$

This induces the Cartan-Eilenberg exact sequence

$$
\begin{aligned}
0 \rightarrow & \operatorname{Hom}(\mathbb{Q} / \mathbb{Z}, C) \rightarrow \operatorname{Hom}(\mathbb{Q} / \mathbb{Z}, \mathcal{D}(C)) \rightarrow \operatorname{Hom}(\mathbb{Q} / \mathbb{Z}, \mathcal{D}(C) / C) \\
& \rightarrow \operatorname{Ext}(\mathbb{Q} / \mathbb{Z}, C) \rightarrow \operatorname{Ext}(\mathbb{Q} / \mathbb{Z}, \mathcal{D}(C)) \rightarrow \operatorname{Ext}(\mathbb{Q} / \mathbb{Z}, \mathcal{D}(C) / C) \rightarrow 0 .
\end{aligned}
$$

Since $\mathcal{D}(C)$ is divisible (= injective) we have $\operatorname{Ext}(\mathbb{Q} / \mathbb{Z}, \mathcal{D}(C))=0$. Also, $C$ is a reduced cotorsion group and thus $\operatorname{Ext}(\mathbb{Q} / \mathbb{Z}, C) \cong C$ by Lemma 2. Moreover, let $A=\operatorname{Hom}(\mathbb{Q} / \mathbb{Z}, \mathcal{D}(C) / C)$. Then the above exact sequence becomes

$$
0 \rightarrow \operatorname{Hom}(\mathbb{Q} / \mathbb{Z}, C) \rightarrow \operatorname{Hom}(\mathbb{Q} / \mathbb{Z}, \mathcal{D}(C)) \rightarrow A \rightarrow C \rightarrow 0,
$$

i.e., $C$ is an epimorphic image of $A$. Therefore it is enough to show that $A$ is an epimorphic image of $P / S$. To do so we determine the structure of $A=\operatorname{Hom}(\mathbb{Q} / \mathbb{Z}, \mathcal{D}(C) / C)$.

First of all we establish the structure of $\mathcal{D}(C) / C$. Clearly, $\mathcal{D}(C) / C$ is both torsion and divisible and so $\mathcal{D}(C) / C \cong \underset{q \text { prime }}{\bigoplus} \mathbb{Z}\left(q^{\infty}\right)^{\left(\gamma_{q}\right)}$, where $\gamma_{q} \leq 2^{\aleph_{0}}$ for each prime $q$ since $|C| \leq 2^{\aleph_{0}}$ by assumption and so also $|\mathcal{D}(C) / C| \leq|\mathcal{D}(C)| \leq 2^{\aleph_{0}}$.

Secondly we note that $\mathbb{Q} / \mathbb{Z} \cong \underset{p \text { prime }}{\bigoplus} \mathbb{Z}\left(p^{\infty}\right)$. It hence follows that

$$
A \cong \operatorname{Hom}\left(\bigoplus_{p \text { prime }} \mathbb{Z}\left(p^{\infty}\right), \underset{q \text { prime }}{\bigoplus} \mathbb{Z}\left(q^{\infty}\right)^{\left(\gamma_{q}\right)}\right) .
$$

This implies, using standard homological facts, that

$$
A \cong \prod_{p \text { prime }} \operatorname{Hom}\left(\mathbb{Z}\left(p^{\infty}\right), \underset{q \text { prime }}{\bigoplus} \mathbb{Z}\left(q^{\infty}\right)^{\left(\gamma_{q}\right)}\right) .
$$

$\operatorname{But} \operatorname{Hom}\left(\mathbb{Z}\left(p^{\infty}\right), \mathbb{Z}\left(q^{\infty}\right)\right)=0$ if $p \neq q$ and so

$$
A \cong \prod_{p \text { prime }} \operatorname{Hom}\left(\mathbb{Z}\left(p^{\infty}\right), \mathbb{Z}\left(p^{\infty}\right)^{\left(\gamma_{q}\right)}\right) .
$$

It is well known — see [4, Proposition 44.3] — that this implies $A \cong \prod_{p \text { prime }} \widehat{J_{p}^{\left(\gamma_{p}\right)}}$ where, as we have seen above, $\gamma_{p} \leq 2^{\aleph_{0}}$ for all 
primes $p$. Therefore $A$ and hence $C$ is an epimorphic image of $P / S=$ $\mathbb{Q}^{\left(2^{\aleph_{0}}\right)} \oplus \prod_{p \text { prime }} \widehat{J_{p}^{\left(2^{\aleph_{0}}\right)}}$, respectively of $P=\mathbb{Z}^{\omega}$.

\section{REFERENCES}

[1] E. Coleman, The Baer-Specker group, Bull. Irish Math. Soc. 40 (1998), 9-23.

[2] M. Dugas and R. Göbel, Endomorphism rings of separable torsion-free Abelian groups, Houston J. Math. 11 (1985), 471-483.

[3] P. Eklof and A. Mekler, Almost Free Modules, Set-theoretic Methods, NorthHolland (1990).

[4] L. Fuchs, Infinite Abelian Groups, I / II, Academic Press (1970/1973).

B. Goldsmith and T. Kelly,

School of Mathematical Sciences,

Dublin Institute of Technology,

Kevin Street,

Dublin 8, Ireland

brendan.goldsmith@dit.ie

tkacfk@hotmail.com

S. L. Wallutis,

FB 6 - Mathematik,

Universität Duisburg-Essen,

45117 Essen,

Germany

simone.wallutis@uni-essen.de

Received on 19 July 2004. 